\title{
Stress: A Pervasive Dilemma in Psychiatric Emergency Care
}

\author{
Paula S. Nurius
}

\begin{abstract}
This paper (1) identifies a number of broad social-situational forces which have had stress producing impact upon the treatment climate and procedures of the psychiatric emergency setting, (2) describes several of the major effects of these factors both on patients and on the emergency unit, (3) applies a person-environment fit (or "mis-fit") perspective in conceptualizing the stress-related problems illustrated by examination of the repeating patient as a high mis-fit group and (4) suggests several recomendations to help increase the "goodness of fit" between the needs, motives and resources of the patient and of the emergency unit and staff
\end{abstract}

$\mathrm{E}$ mergency: urgent necessity; sudden or unexpected occurrence requiring prompt attention; crisis state. Psychiatric emergency cases are, quite literally, defined and characterized by urgent need and stressful conditions. The frequently ambiguous, covert, and complicated nature of the psychiatric component renders these crises all the more difficult to manage and to alleviate. Such cases, perhaps more than any other, would best benefit from an environment which fostered a calm. quiet, thorough exploration of the individual's troubles. The common irony, however, is that the hospital emergency room environment typically operates quite antithetically to this, actually being constituted by a variety of influences which are likely to have a negative impact on patient behavior and patient care.

The characteristic "charged atmosphere" of the emergency setting is most saliently evident in the pervasive sense of time pressure. ${ }^{2}$ Emergency staff put a high premium on "cool speedy care" which translates into assessment, intervention, and disposition being administered as quickly as possible with minimal disruption." While the reasons for this emphasis on speed and brevity are themselves neither trivial nor contrived, the cumulative effect for psychiatric cases is an exacerbation of the patient's condition, ${ }^{4}$ a perception by the clinical staff of their work as "onerous and unrewarding," problem precipitants and dynamics in diagnosis and disposition. ${ }^{\circ}$ Furthermore, the problem tends to fuel itself. That is, the greater are the patients' need states, the more taxed is the limited time resource, the more perfunctory becomes patient care, and the more strained becomes the interpersonal environment for all involved Thus, not only is the presence and effect of debilitating stress in a variety of forms a common dominator across settings, but across treatment provider and recipient as well.

Some of these issues are simply an inherent part of psychiatric emergency care. Evidence indicates, however, that the hospital emergency room as a psychiatric emergency care setting has experienced a rapid and substantial transformation over the past decade and a half. The patchwork approach which has typified efforts to

From the School of Social Work and Institute for Social Research University of Michigan.

Address reprint requests to Paula S. Nurius, Hh.D., 5237 ISK, University of Michigan, Ann Arhor. MI 48109 .

(c) 1984 by Grune \& Stratton, Inc. 0010-440X/84/2503-0012801.00/0 
keep up with changing conditions must now give way to a more consolidated and fundamental reorientation towards treatment. The purpose of this paper is to (1) identify a number of broad social-situational forces which have had significant impact upon the treatment climate of the psychiatric emergency setting, (2) chart the major effects of these factors on the emergency unit, and (3) consider alternatives for remedying the resultant service-related issues and problems.

\section{MAJOR SOCIAL-SITUATIONAL CHANGE FACTORS}

During the past 10 to 15 years, significant qualitative and quantitative changes in the utilization patterns of emergency mental health services have evolved. Not only has there been a rapid growth in the usage of such services, with reported increases as much as $600 \%,{ }^{1.7-11}$ but the nature of the patient population and the manner in which the psychiatric emergency facility is perceived and utilized are also changing. Significant changes in the social and demographic characteristics of individuals using psychiatric emergency services have been widely noted. ${ }^{12-16}$ For example, greater numbers of younger patients ${ }^{17,18}$ as well as an increasing representation of people in lower socioeconomic groups ${ }^{19}$ have been noted. An additional growing trend is the use of emergency facilities in situations other than "true" emergencies. ${ }^{20-23}$

Most importantly, the mental health treatment needs and expectations of the patient population have undergone notable change. Initially, the psychiatric emergency facility was intended to serve primarily an evaluation and referral function for the community, referring psychiatric crisis cases to primary care providers. Emergency facilities now, however, often serve as highly visible and accessible dropin treatment centers as well as entry and re-entry points into the larger mental health system. Their role in management of the severely mentally ill has become increasingly central, frequently as primary caregivers and, at times, as sole sources of treatment. ${ }^{24}$ The emergency facility has in reality if not in plan shifted from serving a triage role to functioning as a source of social support as well as a source of ongoing treatment.

This pervasive trend has been attributed to several social and situational factors, the most broadly influential deriving from the deinstitutionalization movement initiated in the sixties. While the motivating rationale of this reform was to reorient mental health care for the severely and chronically ill toward community-based alternatives, ${ }^{25.26}$ the development or expansion of these alternative treatment sources has seldom been adequate. ${ }^{27}$ The unfortunate consequences of this process has been described in terms of two syndromes (1) "falling between the cracks" due to lack of follow-up or aftercare for discharged patients and (2) "the revolving door" phenomenon wherein individuals cycle in and out of mental health services (particularly inpatient services) with the emergency unit frequently serving as the gobetween. ${ }^{28.29}$

The transformation of the emergency unit during the decade of the seventies was not based on rational, collaborative planning, but rather was largely a response to a system out of control. ${ }^{30}$ With the curtailment of the role of the state hospital, responsibility for the management and disposition needs of the psychotic, the involuntary, and the chronic crisis (repeater) patients in particular has increasingly fallen to the emergency facility. Furthermore, even though most hospitals are ill 
prepared to adequately accommodate these needs, they are increasingly faced with the reality that there is little choice. Adequate substitute provisions were never developed, and of the community centers once available for referral, many have now become saturated. This burden is even further exacerbated by the conflictual treatment priorities and procedures of psychiatric versus medical based emergencies. the resultant strain on medical and nonmedical staff relations, and the subsequent negative effect of these factors on the patient's condition and care. .,7.22.30-32 $^{4}$

There appears also to be a growing expectation that each community center and general hospital will function as a self-sufficient unit offering a range of services appropriate to the needs of their clientele. Interagency relationships have changed such that referral options are often quite limited and constrained. In short, the hospital emergency facility has unwittingly been placed in the role formerly filled by the state hospital, but without the necessary planning, preparation, or resources to even begin to adequately meet the need. Thus, psychiatric emergency services have literally been thrust into the forefront of mental health care, and under decidedly compromising conditions.

\section{MAJOR EFFECTS FELT IN THE EMERGENCY UNIT}

The cumulative effect of these and related factors can best be conceptualized from a person-environment (P-E) fit perspective. Person-environment fit refers to the "goodness of fit" between the characteristics of the person, e.g., motives or abilities, and the properties of the environment, e.g., supply or demand..$^{33,34}$ This perspective maintains that the greater the P-E discrepancy or "mis-fit," the greater the stress and, subsequently, the strain experienced by the individual. Moreover, it is not the nature or level of an environmental demand or supply (or lack thereof) per se that poses a threat to the individual, but rather it is the relative fit or congruence with relevant person variables. This then suggests that efforts toward problem resolution should revolve around enhancement of the person-environment fit.

With respect to the psychiatric patient's experience, P-E mis-fit is exemplified by such contrasts as (1) high need for treatment and/or social support versus low availability of resources; (2) need for a calm, private, reassuring ambiance versus the virtually antithetical characteristics of the emergency room; (3) need for treatment based upon the patient's and his or her support system's competencies and resources versus treatment based on overt behavioral symptoms; (4) need for a view of his or her problems and treatment as legitimate versus the general negative attitude toward psychiatric patients by medical (and sometimes by mental health) staff; (5) need for a sense of trust and rapport with the staff person to encourage compliance and follow-through on treatment recommendations and referrals versus a typically impersonal, detached approach used by staff with virtually no sustained contact or follow-up; and (6) need for adherence to a coherent, sustained treatment plan versus the episodic, uncoordinated quality of treatment characteristic of the revolving door phenomenon.

With respect to the emergency clinician's experience, mis-fit is implicit in such features as (1) the uncertainty and ambiguity which characterizes the information upon which to base decisions; (2) the need for difficult assessments and decisions to be made quickly; (3) the typically chronic nature of work-related tension and 
the high ratio of patients to staff; (4) the consequences of making mistakes are potentially quite serious; (5) the interpersonal contact is emotionally charged, i.e., threatening, exasperating, or worrisome; (6) the patient's problems are often not amenable to treatment through the emergency unit; and (7) the on-site and community-based resources are rarely adequate. For both, the strain of mis-fit is based upon unmet personal needs, taxing environmental demands, unrealistic expectations, and lack of or limited control.

The unchecked outcome of these influences on the psychiatric paticnt is frequently chronicity. A vicious cycle is maintained wherein: patients' needs and abilities seldom adequately mesh with treatment resources and requirements; the motivating problems which were never sufficiently resolved invariably resurface; thus, patients have little choice but to cycle back to their most accessible resource. Unfortunately, of course, the greater the number of patients and their need, the less likely is successful resolution for any, and the more entrenched the cycle becomes.

The eventual outcome for the emergency clinician may well take the form of occupational burn-out. Burn-out refers to a syndrome of emotional exhaustion, depersonalization, and reduced personal accomplishment and satisfaction. ${ }^{33-35}$ The burn-out syndrome is particularly prevalent in high contact "people-work" occupations and its effects can be quite serious for the service provider (in this case, the emergency clinician), the patient, and the institution or unit as a whole. Symptoms of a burning out professional include a cynical and dehumanizing view of patients, a decline in the quality of care provided, greater job dissatisfaction, absenteeism, withdrawal or irritability, and a number of indices of personal dysfunction, e.g., physical exhaustion, illness, increased use of alcohol or drugs, marital and family conflict, and psychological problems. ${ }^{35.36}$

In their research on burn-out, Maslach and her colleagues ${ }^{37-42}$ maintain that the crux of the problem is not interpersonal psychological stress per se, but the social factors involved in provider-recipient relationships and interactions. In terms of the P-E fit framework, burn-out derives from the interaction of person (clinician and patient) and environmental (social-situational) variables. Furthermore, while dispositional, e.g., personality variables are clearly an important factor in burnout, ${ }^{43.44}$ these investigators conclude that the problem is most effectively modified through the social and situational sources of job-related stress. This could range from changes in the organizational structure, protocol, or physical lay-out to flexibility in role definitions, development of professional support networks, and various forms of training or mediation.

\section{ALTERNATIVE CORRECTIVE COUNTERMEASURES}

Recommendations of methods to minimize burn-out tailored to helping professionals have recently begun to emerge. One view (which targets emergency care facilities) holds that the most effective approach is diversification of activities. ${ }^{45}$ The suggested means of operationalizing this approach is through a broad-based, rotational team approach to emergency psychiatric care. Teams would not only be multidisciplinary, but would include individuals from other units within the facility (such as outpatient clinics, inpatient units, consultation-liaison services). This approach presents useful, yet somewhat tenuous possibilities. To avoid the potential pitfalls of multidisciplinary teamwork, for example, careful and collaborative at- 
tention to issues of leadership, staff morale, and clarification of staff functions would be warranted..$^{46}$ Additionally, the goals of diversification would need to be compatible with the goals of promoting follow-up and continuity of carc, of fostering the development and training of expertise in emergency psychiatry, and of stabilizing and enhancing staff relations in the emergency unit.

Research on the most effective coping strategies for the helping and health professional indicates two general strategies (1) getting away from others or (2) turning toward others. ${ }^{39,42}$ To reduce the overload of social and emotional input, planned, temporary social isolation may be effective, e.g., the opportunity to physically or psychologically withdraw or to shift to tasks that do not involve contact with people. The latter approach, on the other hand, would involve use of one's social-professional support systems, ideally with active support and facilitation by the larger institution. Peers and colleagues have been found to be potentially valuable sources of information, comfort, affirmation, encouragement, and reflection.

By and large, these remedies approach the problem via means of buffering or directly reducing the effects of existing stressful conditions. Cognizance of the problem and experimentation with various coping strategies will no doubt reduce strain and, thus, enhance service provision. An important complement to such strategies would he to examine the appropriateness of the fundamental model undergirding emergency psychiatric care as it has evolved today. All evidence indicates that the evaluation and referral model is no longer viable. Instead. psychiatric emergency services must now undergo a restructuring of its resources and its orientation toward treatment to be consistent with a crisis resolution model. To more fully explore this alternative, study findings regarding a patient group representing a particularly high degree of mis-fit (the chronic crisis or repeater patient) will be considered.

\section{CHARACTERISTICS OF THE REPEATER PATIENT}

One practical means of conceptualizing the mis-fit dimension of chronicity is in terms of its "costliness." The cost borne by the patient, for example, is frequently in the form of enduring negative attitudes and reactions as well as chronically unresolved problems. The emergency medical staff tend to view this patient group as inappropriate, disruptive, and time and energy costly. For the mental health staff, this group perhaps more than any other represents their sense of futility and frustration-standing as a reminder of the limits of the clinician's ability to help. Therefore, the mental health staff too must continually struggle with the question of how much of their time, energy, and resources to invest in the repeater patient. Consequently, rightly or wrongly, the dimension of time has become a focal point of contention in assessing the appropriateness and worthiness of this patient group.

Recent research" provides one perspective on this issue of time cost (and the problems it directly and indirectly reflects). Two measures were used to capture the critical time dimension (1) the amount of time the psychiatric patient spent in the emergency room per visit and (2) the number of times per year a patient utilized emergency psychiatric services. The latter measure of chronicity was examined in two ways. One variable differentiated the patient who visited the emergency unit one to three times a year (non-repeater) from those with four or more visits 
(repeater). This distinction is more conservative and perhaps more meaningful as a demarcation of chronicity or overutilization than the single versus multiple episodes distinction frequently used to delineate the patient population. The second variable breaks down the repeater group and more finely distinguishes all patients by the number of treatments episodes they had in a given year, e.g., (1) 1 to 3, (2) 4 to $6,(3) 7$ to $11,(4) 12$ to $20,(5)$ more than 20.

\section{Time Per Visit}

By and large, an overall lack of significance was found in tests (chi-square, $P<$ .05) involving time in the emergency room and patient personal (social/demographic) and clinical (diagnosis, presenting problem, disposition) characteristics. One noteworthy exception was the association of greater time needs with the case disposition of psychiatric hospitalization. Patients requiring hospitalization spent significantly more time in the emergency room, but repeaters were neither significantly more likely to be hospitalized nor did they spend more time per visit in the emergency room than non-repeaters.

It would appear that factors other than those directly attributable to the patient measured here would account for the variation in time per visit. One important and direct source of influence to consider is the manner in which the emergency facility processes psychiatric patients, both the repeater and the non-repeater. This is a crucial alternative hypothesis to consider and one supported elsewhere. ${ }^{24.30}$ If indeed much of the time dimension is associated with variables beyond the patient's control, e.g., triage system, treatment protocols, patient load, reluctance by staff to respond, then time efficiency solutions should be considerably easier to institute.

For example, many facilities rely on lock-step protocols geared primarily toward flow control of large numbers of people and toward treatment of physical problems. Such systems commonly funnel all patients through the same intake procedures, house them in noisy, congested waited areas, and require examination by medical personnel even if the presenting problem is nonmedical in nature. For the patient with acute psychiatric distress, this environment represents a painful lack of fit with his or her need. An alternative system could prioritize careful collection of specific information about the patient at intake to better determine what his or her primary needs are. For those primarily requiring psychiatric intervention, attempts could be made to create a separate quieter, more controlled environment in which they could wait, be observed, and be interviewed. Intervention by medical personnel could be presented as an available service rather than a prerequisite for those with nonmedical presenting problems. Additionally, clear criteria for psychiatric triage should be developed to better determine the nature of the patients' treatment needs and, thus, which psychiatric staff person would be best suited to meeting their needs.

\section{Chronicity}

In contrast to the prior time measure, the measures of chronicity indicated numerous marked differences between those with high versus low service utilization patterns. Repeaters were significantly more likely to be male, single, unemployed, and indigent and were significantly less likely to require medical attention. Repeaters were much more likely to receive a diagnosis in the psychosis category (schizophrenia 
in particular) and were less likely to be diagnosed as neurotic or suffering from a transient situational disturbance. The problems presented by the two groups also tended to be quite different. Repeaters, for example, were more likely to report problems associated with psychosis and with suicidal ideation. They were also more likely to request psychiatric hospitalization and to need assistance with other types of referral or placement planning.

The number of episodes variable was useful in allowing for comparisons within the repcater population. The most frequent repeaters were most likely to be male, unemployed, alone, and least likely to be privately insured or supported. The most frequent repeaters were also more likely to receive disorder diagnosis such as schizophrenia or personality disorder and less likely to be diagnosed as having a neurotic or transient disturbance. Among the presenting problems and dispositions, repeaters of various episode levels differed, but typically not in systematic ways. It would appear that repeaters are not only reliably different from non-repeaters in various domains, but are also heterogenous in several respects among themselves.

In stark contrast to these notable differences in personal and clinical characteristics between the repeating and nonrepeating groups is the total lack of significant differences in case outcomes. There is no way of ascertaining from these data whether the nature of the intervention provided was qualitatively different for the two groups or not. Yet the administration of treatment as reflected by time spent, likelihood of hospitalization, and the nature of disposition and referral evidenced no systematic differences. To the extent that these variables capture the resources afforded by the emergency room environment, the lack of fit with different patient needs and motives is clear. To be sure, this troublesome circumstance is in some part due to the all too frequent dearth of resources a vailable to the emergency unit, e.g., insufficient staff, limited community resources, strained interagency resources. Thus, once again, both patient and staff are found subject to the phenomenon of mis-fit with almost certain stress and strain.

An obvious change suggested by these findings involves greater differentiation of treatment, disposition, and referrals provided to different types of patients. Staff should be supported, for example, in making more thorough and careful assessments of the precipitants and dynamics underlying patient problems and to their individual capabilities and resources (or lack thereof). For instance, factors commonly associated with social isolation, poverty, and chronic disorders were found in the reported sample to be characteristics of those paticnts rcturning repeatedly. Social and personal conditions such as these constitute powerful unmet needs which greatly influence the patient's perceptions and behavior. Such patients would benefit from assistance in first perceiving alternative sources of aid, e.g., of goods, treatment, support, and then in learning how to effectively utilize these sources. This may well require more active consultation and collaboration among facilities in the referral network as well as greater contact with individuals personally related to the patient. Without such assistance, high mis-fit patients are likely to continue in the pattern of overextending their most familiar, visible, and available resourcethe emergency facility.

As noted earlicr, staff nceds and issues also need to be directly addressed. To some extent, a reorientation toward treatment which focuses on more effective, less pressured, and more fulfilling interactions between patient and staff should serve 
to reduce stress and strain. However, a variety of contingencies for support and problem resolution should also be considered. Opportunities for structured discussion and sharing among staff, and about patients, work issues, and suggestions for change, for example, could be provided both to negotiate solutions and to foster cohesion and morale. Importantly, such exchanges should not be limited to the mental health staff as a group, but should instead draw medical and mental health personnel together as a collective.

\section{CONCLUSION}

In sum, this paper has identified a number of major changes in utilization patterns of emergency psychiatric care services over the past decade and a half. Broad socialsituational forces associated with these changes have been noted and discussed in relation to their impact on the treatment climate and procedures of the emergency care unit. A person-environment (P-E) fit or "mis-fit" perspective has been offered as a useful and constructive framework to use in conceptualizing the evolving problems and deficiencies. The general relationship of P-E fit to stress and felt strain has been explored and has been illustrated by examination of a high mis-fit group-the chronic or repeating patient. Finally, several recommendations have been made to help increase the "goodness of fit" between the needs, motives, and resources of the patient as person and of the emergency unit and staff as environment.

\section{REFERENCES}

1. Spitz, L: The evolution of a psychiatric crisis intervention service in a medical emergency room service. Comp Psychiatr 17:99-113, 1976

2. Bartolucci, G, Drayer, CS: An overview of crisis intervention in the emergency rooms of general hospitals. Am J Psychiatr 130:953-960, 1973

3. Roth, J: Some contingencies of the moral evaluation and control of clientele. Am J Soc $77: 839,1972$

4. Errera, P, Wyshak, G, Jarecki, H: Psychiatric care in a general hospital emergency room. Arch Gen Psychiatr 9:105-112, 1963

5. Blane, H, Muller, J, Chafetz, M: Acute psychiatric service in the general hospital: II. Current status of emergency psychiatric services. Am J Psychiatr October (suppl)37-45, 1967

6. Rusk. T: Psychiatric education in the emergency room setting. Can Psychiatr Assoc J 16:111-120, 1971

7. Coleman, JV: Research in walk-in psychiatric services in general Hospitals. Am J Psychiatr 124:1668-1673, 1968

8. Frankel, F, Chafetz, M, Blane H: Treatment of psychosocial crises in the emergency service of a general hospital. JAMA, 195:114-116, 1966

9. Jacobson, G: Emergency services in community mental health. Am J Public Health, 64:124-128, 1974

10. Witkin, M: Emergency Services in Psychiatric Facilities. National Institute of Mental Health Statistical Note 136, Rockland, Md., NIMH, 1977

11. Nurius, PS: Emergency psychiatric services: A study of changing utilization patterns and issues. Int J Psychiatr Med 13:239-254, 1983

12. Huffine, C, Craig, T: Social factors in the utilization of an urban psychiatric emergency service. Arch Gen Psychiatr 30:249-255, 1974

13. Munoz, R, Tuason, VB, Dick, E: The psychiatric room service patterns. Comp Psychiatr 11:185-189, 1970

14. Satloff, A, Worby, C: The psychiatric emergency service: Mirror of change. Am J Psychiatr 126:1628-1632, 1970 
15. Slaby, AE, Lieb, J, Tancredi, LR: Handbook of Psychiatric Emergencies, Medical Examination Publishing Co., Flushing, New York, 1975

16. Zonana, H, Henisz, JE, I evine, N: Psychiatric emergency services a decade later. Int J Psychiatr Med 4:273-289, 1973

17. Sandifer, Jr, MG: Science and set in treatment decisions, Am J Psychiatr 128:11401145,1972

18. Simmons, J: The psychiatric emergency in adolescence. Int Psychiatr Clin 3:37-51, 1966

19. Linn, L: Emergency room psychiatry: A gateway to community medicine. Mt Sinai J Med 38:110-120, 1971

20. Trier, T, Levy, R: Emergent, urgent, and elective admissions. Arch Gen Psychiatr $21: 423-430,1969$

21. Wayne, G: The psychiatric emergency: An overview, in Wayne, G, Koegler, R (eds):

Emergency Psychiatry and Brief Therapy. Boston, Little, Brown and Co., 1966

22. Grumet, W, Trachtman, G: Psychiatric social workers in the emergency department.

Health Social Work 1:114-131, 1976

23. Slaby, AE, Perry, PL: Use and abuse of psychiatric emergency services. Int J Psychiatr Med 10:1-8, 1980

24. Gerson, S, Bassuk, E: Psychiatric emergencies: An overview. Am J Psychiatr 137:111,1980

25. Joint Commission on Mental Health and Mental Illness: Action for Mental Ilealth.

New York, Basic Books, 1961

26. Bassuk, EL, Gerson, S: Deinstitutionalization and mental health services. Sci Am 238:46-53. 1978

27. Slovenko, R, Luby, E: From moral treatment to railroading out of the mental hospital

Bull Am Acad Psychiatr Law, 2:223-236, 1974

28. Johnson, J: Psychiatric emergencies in the community. Comp Psychiatr 10:275-284, 1969

29. Talbott, JA: Deinstitutionalization: Avoiding the disasters of the past. Hospital Commun Psychiatr 30:621-624, 1979

30. Bassuk, E, Schoonover, S: The private general hospital's psychiatric emergency service in a decade of transition. Hospital Commun Psychiatr 32:181-185, 1981

31. Barton, GM: A hospital's political environment and its effect on the patient's admission. Hospital Commun Psychiatr 25:156-169, 1974

32. Beahan, L: Emergency mental health services in a general hospital. Hospital Commun Psychiatr 21:81-84, 1970

33. French, Jr, JPR, Rogers, W, Cobb, S: Adjustment as person-environment fit. In Coelho, GV, Hamburg, DA, Adams JE (eds): Coping and adaptation. New York, Basic Books, 1974

34. Harrison, V. Person-environment fit and job stress, in Cooper, CL, R. Payne (eds): Stress at work. New York, Wiley, 1978

35. Freudenberger, HJ: Staff burn-out. J Social Issues 30:159-165. 1974

36. Maslach, C: Burned-out. Human Behavior 5:16-22, 1976

37. Maslach, C, Jackson, SE: Burnout in health professions: A social psychological analysis, in Sanders, GS, Suls, J (eds): Social Psychology of health and illness. Hillsdale, N. J., Erlbaum, 1982

38. Maslach, C: The burn-out syndrome and patient care, in Garfield, C (ed); Stress and Survival. St. Louis, Mosby, 1979

39. Jackson, SE, Maslach, C: After-effects of job-related stress: Families as victims. J

Occupational Behavior 3:63-77, 1982

40. Maslach, C: The client role in staff burn-out. J Social Issues 34:111-124, 1978

41. Maslach, C, Jackson, SE: The measurement of experienced burnout. J Occupational Behavior 2:99-113, 1981

42. Pines, A, Maslach, C: Characteristics of staff burn-out in mental health settings. Hospital Commun Psychiatr 29:233-237, 1978

43. Gann, ML: The role of personality factors and job characteristics in burnout: A study 
of social service workers. Unpublished doctoral dissertation. University of California, Berkeley, 1979

44. Heckman, SJ: Effects of work setting, theoretical orientation, and personality on psychotherapist burnout. Unpublished doctoral dissertation. California School of Professional Psychology, Berkeley, 1980

45. Slaby, AE: Emergency psychiatry: An update. Hospital Commun Psychiatr 10:687698, 1981

46. Gomez, EA, Ruiz, P, Langrod, J: Multidisciplinary team malfunctioning on a state hospital unit: A case study. Hospital Commun Psychiatr 31:38-40, 1980 